\title{
Lasing cholesteric capsules
}

\author{
${ }^{1}$ Dudok T. H., ${ }^{1}$ Savaryn V. I., ${ }^{2}$ Meyer C., ${ }^{3}$ Cherpak V. V., ${ }^{3}$ Fechan A. V., \\ ${ }^{4}$ Lychkovskyy E. I., ${ }^{5}$ Pansu B. and ${ }^{1}$ Nastishin Yu. A. \\ ${ }^{1}$ Vlokh Institute of Physical Optics, 23 Dragomanov Street, 79005 Lviv, Ukraine \\ ${ }^{2}$ Laboratoire de Physique des Systemes Complexes, Universite de Picardie, Jules \\ Verne, 33 rue Saint-Leu, 80039 Amiens, France \\ ${ }^{3}$ Lviv Polytechnic National University, S. Bandera Street, 12, 79013 Lviv, Ukraine \\ ${ }^{4}$ Lviv Danylo Halytsky National Medical University, Pekarska Street, 69, 79010 \\ Lviv, Ukraine \\ ${ }^{5}$ Laboratoire de Physique des Solides, UMR 8502, Universite Paris-Sud, 91405 \\ Orsay, France
}

Received: 02.08 .2016

\begin{abstract}
We describe a compact point laser source based on dye-doped cholesteric encapsulated in a flexible polymer film. Optically pumped emission spectra of the capsule are experimentally studied.
\end{abstract}

Keywords: mirrorless lasing, photonic bandgap, liquid crystal lasers

PACS: $42.55 . \mathrm{Zz}$

UDC: $538.958+681.7 .069 .24$

\section{Introduction}

There are several mechanisms of mirrorless laser generation in the media with the structure or geometry providing optical feedback. The important examples are laser generations in the scattering media (or random lasing), the photonic crystals (or photonic bandgap-edge lasing) and the closed microcavities (whispering gallery mode lasing), as well as the generation using the nanoparticles of noble metals, which employs a mechanism of surface plasmon resonance (lasing with the optical feedback of the type referred to as a 'surface plasmon amplification by stimulated emission of radiation', or simply SPASER) [1].

Lasing that occurs in cholesteric liquid crystals is associated with mirrorless resonant light amplification in self-assembled photonic structures. It has been shown unambiguously [2] that one or two lasing lines can be detected in flat cholesteric cells with planar molecular alignment. They appear on the substrates at one or both edges of a cholesteric photonic bandgap (PhBG), if the latter gap overlaps with the dye-fluorescence emission band. Notice that the PhBG represents a distinguishing property of any photonic structure that provides inhibition of light emission within the spectral region of the $\mathrm{PhBG}$ and strong leaking of the emission at its edges. The latter implies that the density of states for the emitted photons vanishes inside the PhBG but diverges at its edges. It is believed that the perfection of planar alignment of the cholesteric layers is a key requirement for the laser generation in this medium.

Our recent experiments [3] have revealed that cholesteric-alignment imperfections manifest themselves in the lasing spectra. Moreover, they have demonstrated that the laser generation in cholesterics can be obtained not only in well-aligned, thin planar cells - which is a common view in the current literature, - but also in thick defective cells with untreated though flat substrates. 
The goal of the present work is to check the possibilities for the laser generation in the containers with essentially non-flat shapes such as plastic capsules. If indeed possible, such lasers could, in principle, serve as point light sources with technologically simple fabrication. In particular, we are going to examine the laser generation in a plastic capsule filled with a fluorescent-dye-doped cholesteric.

\section{Experimental}

To fabricate plastic capsules, we used a commercially available polymer, polyethylene terephthalate. It is sometimes called as poly(ethylene terephthalate), being also known as Dacron, Terylene or Lwsan. Its common abbreviation is PET or PETE (PETP or PET-P in older literature sources). A capsule was obtained by forming cavities in a PET film by means of a thermal vacuum technique [4]. With this technique, a thermally softened plastic sheet is vacuum-sucked in a mould that fits its shape.

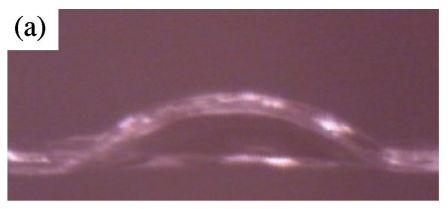

(c)

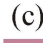

(b)
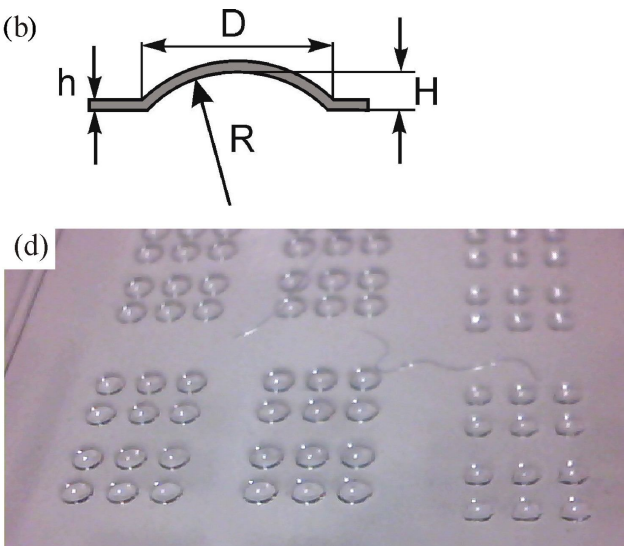

Fig. 1. A cavity in a plastic sheet, which is filled with a cholesteric laser mixture and forms a cholesteric capsule: a side-view photograph of the cavity (a), a sketch that introduces the sizes of the latter, $h=0.145 \mathrm{~mm}$, $H=0.32 \mathrm{~mm}, D=1.8 \mathrm{~mm}$, and $R=2.5 \mathrm{~mm}(\mathrm{~b})$, a top-view photograph of the cavity (c), and a matrix of cavities forming the cholesteric capsules (d).

The cavities in the plastic sheet were filled with a cholesteric mixture KET $90700+21 \% E 7+1 \% \mathrm{NR}$, which was studied in details in Ref. [3]. After filling the cavities, the plastic sheet was glued to a glass substrate. In this way one can fabricate a matrix of dot light sources shown in Fig. 1d, such that the sizes of the cavities and the distances between them can be taken as small as tens of micrometers.

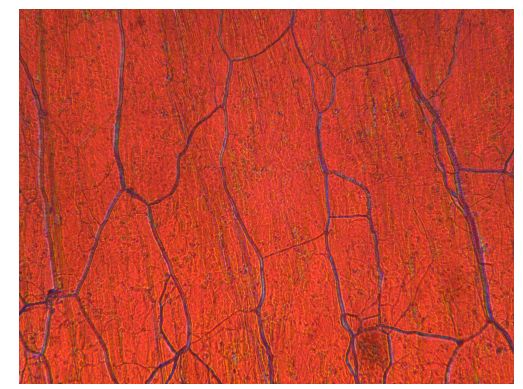

Fig. 2. Optical polarization microscopy texture of cholesteric KET90700+21\%E7+1\%NR in glass cells with the $100 \mu \mathrm{m}$ gap.

Because of relatively high thickness $(H=0.32 \mathrm{~mm})$ and shape curvature of the capsules, the polarization microscopy textures become opaque, being significantly affected by the light scattering inside the capsules and the refraction at their curved surfaces. Nonetheless, it is easily under- 
stood that the bulk cholesteric structure is grainy defective. The defects of the cholesteric structure accessible for optical microscopy observations in thinner flat cells are so-called 'oily streaks' (see Fig. 2) and focal conical domains [5], as well as dislocations and disclinations. A densely defective cholesteric structure in the thick samples, including that of the capsules under test, produce strong light scattering and, consequently, the conditions necessary for the random lasing [3].

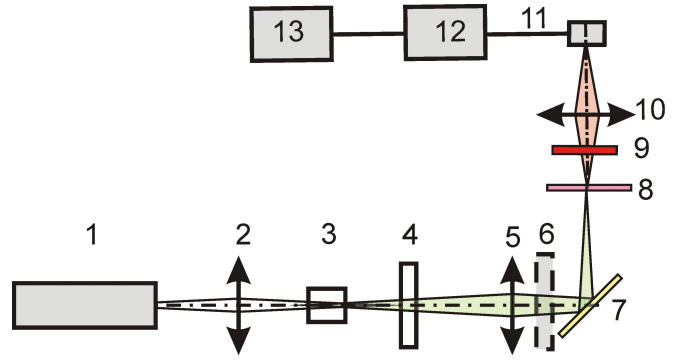

Fig. 3. Optical scheme of experimental setup for the spectral studies of laser generation from a dyedoped cholesteric liquid crystal: 1 - pulse solidstate laser $(\lambda=1064 \mathrm{~nm}), 2,5$ and $10-$ focusing lenses, 3 - second-harmonic generating material, 4, 6 and 9 - optical filters, 7 - rotating mirror, 8 - sample under study, 11 - optical fibre cable, 12 - fibre spectrometer, and 13 - computer.

The emission spectra were measured using an experimental setup shown in Fig. 3. Laser generation from the capsules shown in Fig. 1 was measured in the two geometries, from the flat side of the glass substrate and from the curved plastic surface of the capsule.

\section{Experimental results and discussion}

The emission spectra measured in the two geometries mentioned above are shown in Fig. 4. The spectral signals from the flat (curve 1 in Fig. 4) and curved (curve 2 in Fig. 4) sides of the capsule are not identical. The emission band detected from the curved capsule surface is clearly narrower than that from the flat surface. The locations of these emission bands suggest that the band originated from the flat side appears wider because it includes the emission band from the curved side. Decomposition of the bands into Gaussian peaks followed by overlap with the PhBG spectrum (which is the reflection spectrum for a planarly aligned cholesteric) reveals that, among the Gaussians constituting the emission band measured, there are both 'forbidden' and 'allowed' PhBG lines (see Fig. 5 and Fig. 6).

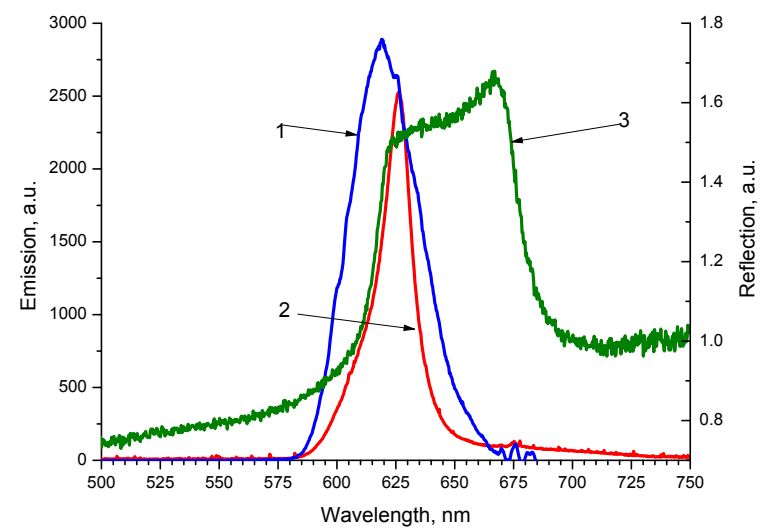

Fig. 4. Emission spectra of cholesteric capsules detected from the flat (curve 1 , blue) and curved (curve 2, red) surfaces These spectra are superimposed with the selective reflection spectrum (curve 3 ) of the mixture KET90700+21\%E7+1\%NR (see Ref. [3]).

Following from the classification given in Ref. [3], the 'allowed' $\mathrm{PhBG}$ lines are those located at the external sides of the PhBG edges, whereas the 'forbidden' lines fall inside the PhBG. One can see from Fig. 5 that the constituent Gaussian lines peaked at 626.1 and $632.0 \mathrm{~nm}$ are forbidden and those located at 600.4, 611.6 and $619.55 \mathrm{~nm}$ are allowed. Of course, the only line present at $619.5 \mathrm{~nm}$ for a thin, well aligned cholesteric cell must be classified as the allowed one. Then the other lines located at 600.4 and $611.6 \mathrm{~nm}$ should be explained by a 'blue' spectral shift of 
the PhBG occurring for the tilted orientations of the cholesteric layers with respect to the cell normal, as well as by modification of the fine structure of the PhBG spectrum in the presence of structural distortions. Here the 'blue' spectral shift means a shift of the PhBG towards shorter wavelengths for the tilted viewing angles. According to Ref. [6], this leads to a corresponding hypsochromic shift of the PhBG lasing lines.
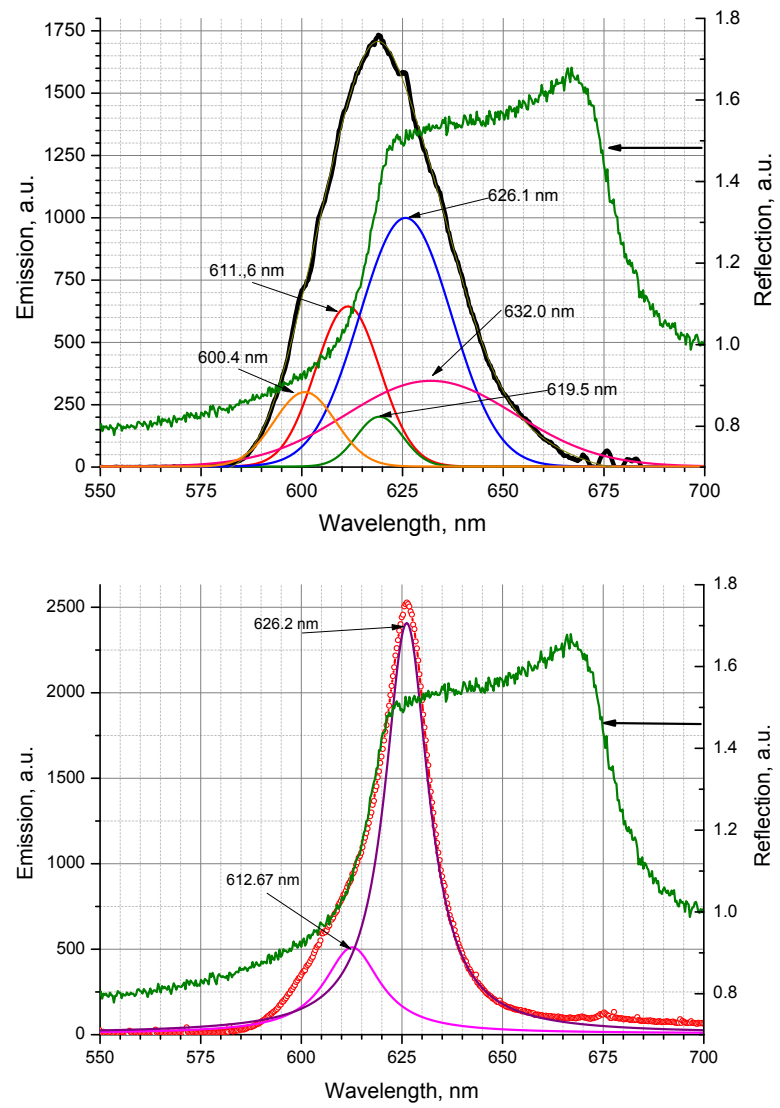

Fig. 5. Emission spectrum measured from the flat side of the cholesteric capsule (shown by curve (1) in Fig. 4) and its decomposition into constituent Gaussian spectral peaks centred at $600.4,611.6$, $619.5,626.1$ and $632.0 \mathrm{~nm}$. This spectrum is superimposed with the cholesteric PhBG spectrum of the mixture KET90700+21\%E7+1\%NR.

Fig. 6. Gaussian decomposition of the emission spectrum detected from the curved capsule side, shown by curve 2 in Fig. 4, resulting in the peaks centred at 612.67 and $626.2 \mathrm{~nm}$ with respect to the cholesteric PhBG spectrum of the mixture KET $90700+21 \% E 7+1 \%$ NR.

Concerning the fine structure of the PhBG wings, it is worth noting that the spectrum has an oscillation character on the both wings of the PhBG, similar to what is schematically shown in Fig. 7. Such a fine structure can be easily detected in well aligned thin cells [7]. One can expect that the fine structure in distorted, defective samples is also imperfect, such that the oscillation maximums do not necessarily decrease in their amplitude on departing from the PhBG edge, acquiring instead a random character. In such a case the laser generation could be observed at any spectral position at the PhBG wing. However, there is no theoretical or experimental data in the current literature concerning the influence of structural defects on the shape of the PhBG, especially for densely defective cholesterics. Moreover, the techniques applied usually for experimental determination of the $\mathrm{PhBG}$ from the reflection spectra might prove inapplicable for the densely defective cholesteric samples, since the measured light signal is then integrated over the area with significantly nonuniform director distribution. Instead, one needs a technique for measuring the reflection spectra that scans a sample point by point, which is akin to a scanning confocal microscopy.

In a similar manner, a standard Gaussian decomposition can be applied to the emission spectrum detected from the curved side of our cholesteric capsule (see curve 2 in Fig. 4). It follows 
from Fig. 6 that the line located at $612.67 \mathrm{~nm}$ is allowed. Most probably, it is affected by a 'blue' shift or/and belongs to the fine structure of the short-wavelength wing of the PhBG.

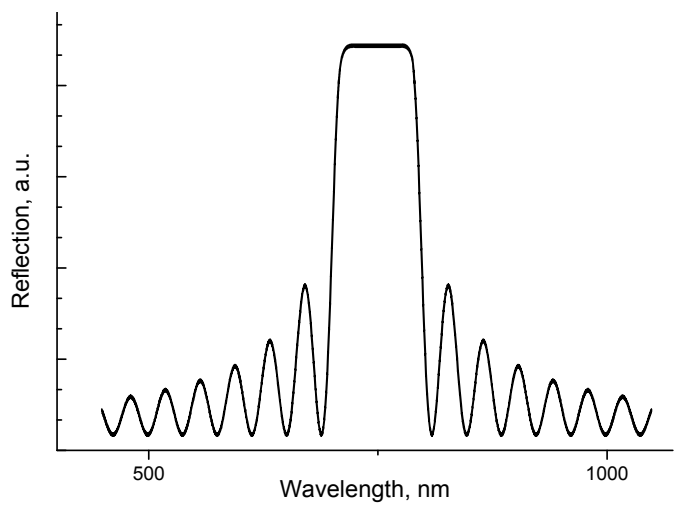

Fig. 7. Schematic form of the reflection spectrum with oscillations appearing at the PhBG wings.

It is interesting that the emission spectra decomposed from the flat and curved sides contain the constituent lines located at quite close wavelengths. These are $611.6 \mathrm{~nm}$ in Fig. 5 versus $612.67 \mathrm{~nm}$ in Fig. 6, and $626.1 \mathrm{~nm}$ in Fig. 5 versus $626.2 \mathrm{~nm}$ in Fig. 6. Most probably, this fact implies the same nature of the lines in each pair and the differences between different pairs of lines.

We have compared the emission spectra obtained from the curved capsule side (see curve 2 in Fig. 4) to those observed for a $100 \mu \mathrm{m}$ cell with smooth glass substrates [3]. It is seen from Fig. 8 that the decomposed emission spectrum for the cholesteric capsule contains a peak located at $612.67 \mathrm{~nm}$ (curve 2b), which can be classified as the allowed PhBG line. On the other hand, this line is absent in the spectrum measured for the flat $100 \mu \mathrm{m}$ glass cell. The second line available at $626.2 \mathrm{~nm}$ for the cholesteric capsule covers the both lines found for the flat glass cell (620.24 and $631.9 \mathrm{~nm}$ ). Therefore, both the allowed and the forbidden lines are detected, in common for the two geometries under test.

Since the cholesteric capsules are rather thick, being formed with no special treatment of the surface, cholesteric pseudo-layers are significantly distorted and essentially curved, with high number of structural imperfections included. As a consequence, they scatter efficiently the emitted light. Then the emission bands contain the constituent Gaussians of the same origin, i.e. the forbidden lines typical for thick defective flat cells. Hence, the light amplification by its nature can be classified as the random lasing.

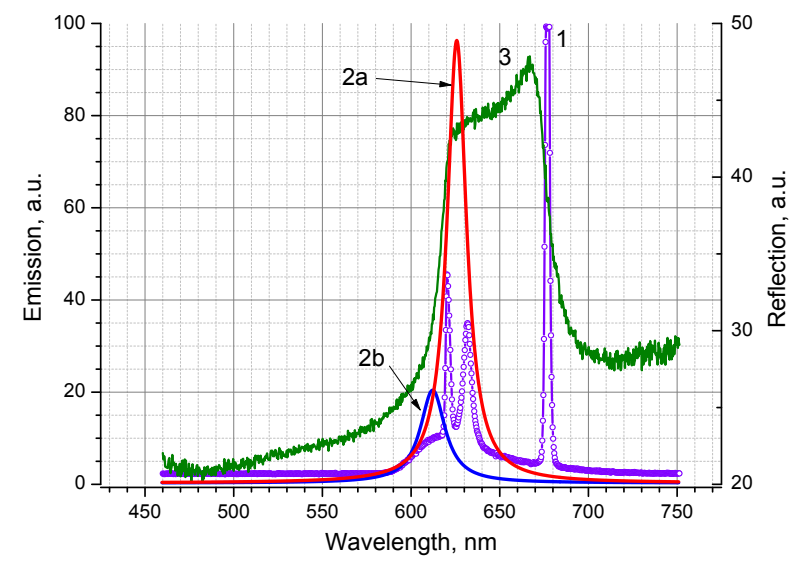

Fig. 8. Light emission spectrum of our cholesteric capsule. The spectrum is decomposed into two peaks (curves $2 \mathrm{a}$ and $2 \mathrm{~b})$ overlapped with the emission spectrum for the $100 \mu \mathrm{m}$-thick glass cell (curve 1) and the selective reflection band (curve 3 ) of the mixture KET90700+21\%E7+1\%NR. 
The difference between the two sets of the constituents for the decomposed spectra measured from the curved and flat surfaces of our capsule can be assigned to the difference in the orientations of cholesteric layers. Relative narrowness of the emission band detected from the curved side suggests that the alignment of the cholesteric layers at the interface with the polymer film is rather planar, i.e. parallel to the surface. The results presented in Ref. [8] indicate that thermal extrusion of the plastic films induces a well pronounced orientation effect for the polymer chains along the direction of the film extrusion. The liquid crystal molecules become aligned along this direction due to intermolecular forces. However, in our opinion, the geometrical factor is more important. Namely, this can be a wave-like profile of the film surface, which can be expected under thermal stretching of the film. It is followed by elastic relaxation that occurs during cooling the sample down to the room temperature and is accompanied by a negative pressure. As a result, it is probable that the film surface has a wavy profile on a nanoscale. The geometrical profile that cancels degeneration along the tangential orientation of the director represents a much stronger factor, when compared to the intermolecular forces and the entropy effects of excluded volume [8]. This also agrees with a higher quality of the planar alignment of cholesteric liquid crystal molecules at the polymer film surface. Concerning the flat surface, it is worth noting that the glass substrates have not been treated and so they allow for any azimuthal orientation of the director, including highly defective ones.

It remains to explain the differences between the emission spectra of thick, highly imperfectly aligned planar cells studied in details in Ref. [3] and the cholesteric capsules studied here. The most evident structural difference between the two geometries is that in the case of thick though highly defective cells, the planar orientation, at which the cholesteric layers are parallel to the surface, dominates. In the case of capsules, one expects a considerably richer set of orientations deeply in the bulk of the capsule, from planar to normal (with respect to the surface). This is due to much larger thickness of the capsule and topological constrains related to its curvature. Tilted orientation, with respect to the light propagation direction, of the cholesteric layers located deeply in the bulk can lead to a hypsochromic shift of the selective reflection wavelengths and, hence, to a shift in the Bragg amplification of the emitted light [6].

\section{Conclusions}

We have fabricated matrices of dot laser sources based on capsulation of a dye-doped cholesteric in a periodical array of cavities placed in a polymer sheet. We have found that optically pumped emission spectra of these capsules contain laser-like peaks, which are close to those observed in imperfectly aligned thick cholesteric cells. The laser-like peaks or, more precisely, the constituent laser lines composing these peaks are located both at the short-wavelength edge of the PhBG (socalled 'allowed' lines) and inside the PhBG (so-called 'forbidden' lines). We have concluded that the matrices of cholesteric capsules can turn out to be promising for fabricating dot laser sources based on dye-doped cholesterics.

\section{Acknowledgement}

This publication is supported by the Ministry of Education and Science of Ukraine.

\section{References}

1. Nastishin Yu A and Dudok T H. Optically pumped mirrorless lasing. Lviv: Publishing House of Vlokh Institute of Physical Optics (2015).

2. Kopp V I, Fan B, Vithana H K M and Genack A Z, 1998. Low-threshold lasing at the edge of a 
photonic stop band in cholesteric liquid crystals. Opt. Lett. 23: 1707-1709.

3. Dudok TH, Savaryn V I, Krupych O M, Fechan A V, Lychkovskyy E, Cherpak V V, Pansu B, and Nastishin Yu A, 2015 Lasing in imperfectly aligned cholesterics. Appl. Opt. 54: 9644-9653.

4. Throne J L. Understanding thermoforming. Munich: Carl Hanser Verlag (2008).

5. Kleman M, Lavrentovich $\mathrm{O} D$ and Nastishin $\mathrm{Yu}$ A. Dislocation and disclination in mesomorphic phases. In: Dislocations in solids. Vol. 12. Ed. by F R N Nabarro and J P Hirth. Elsevier (2004). p. 147-271.

6. Palto S P, Shtykov N M, Umansky B A, Barnik M I and Blinov L M, 2006. General properties of lasing effect in cholesteric liquid crystals. Opto-Electron. Rev. 14: 323-328.

7. Abdulhalim I, Benguigui L and Weil R, 1985. Selective reaction by helicoidal liquid crystals. Results of an exact calculation using the $4 \times 4$ characteristic matrix method. J. Physique. 46: 815825.

8. Stetsyshyn Y, Kostruba A, Harhay K, Donchak V, Ohar H, Savaryn V, Kulyk B, Ripak L and Nastishin Yu A, 2015. Multifunctional cholesterol-based peroxide for modification of aminoterminated surfaces: Synthesis, structure and characterization of grafted layer. Appl. Surf. Sci. 347: 299-306.

Dudok T.H., Savaryn V.I., Meyer C., Cherpak V.V., Fechan A.V., Lychkovskyy E.I., Pansu B. and Nastishin Yu.A. 2016. Lasing cholesteric capsules. Ukr.J.Phys.Opt. 17: 169 - 175

Анотація. Описано компактне гнучке точкове лазерне джерело, створене иляхом капсулювання забарвленого флуоресиентним барвником холестерика в полімерну плівку. Досліджено спектри емісії ичих капсул під дією оптичного збудження. 